\title{
Inflammation, stress, and diabetes
}

\author{
Kathryn E. Wellen and Gökhan S. Hotamisligil
}

Department of Genetics \& Complex Diseases, Harvard School of Public Health, Boston, Massachusetts, USA.

\begin{abstract}
Over the last decade, an abundance of evidence has emerged demonstrating a close link between metabolism and immunity. It is now clear that obesity is associated with a state of chronic low-level inflammation. In this article, we discuss the molecular and cellular underpinnings of obesity-induced inflammation and the signaling pathways at the intersection of metabolism and inflammation that contribute to diabetes. We also consider mechanisms through which the inflammatory response may be initiated and discuss the reasons for the inflammatory response in obesity. We put forth for consideration some hypotheses regarding important unanswered questions in the field and suggest a model for the integration of inflammatory and metabolic pathways in metabolic disease.
\end{abstract}

\section{Inflammation, stress, and diabetes}

Survival of multicellular organisms depends on the ability to fight infection and heal damage and the ability to store energy for times of low nutrient availability or high energy need. Metabolic and immune systems are therefore among the most basic requirements across the animal kingdom, and many nutrient and pathogen-sensing systems have been highly conserved from organisms such as Caenorhabditis elegans and Drosophila to mammals. Perhaps not surprisingly, metabolic and immune pathways have also evolved to be closely linked and interdependent. Many hormones, cytokines, signaling proteins, transcription factors, and bioactive lipids can function in both metabolic and immune roles. In addition to using some of the same cellular machinery, metabolic and immune systems also regulate each other. The normal inflammatory response relies upon metabolic support, and energy redistribution, particularly the mobilization of stored lipid, plays an important role in fighting infection during the acute-phase response (1). The basic inflammatory response thus favors a catabolic state and suppresses anabolic pathways, such as the highly conserved and powerful insulin signaling pathway.

The integration of metabolism and immunity, which under normal conditions is beneficial for the maintenance of good health, can become deleterious under conditions of metabolic challenge, as exemplified by the immunosuppression characteristic of malnourished or starving individuals (1-3). Famine has been a prominent hazard to human health throughout history, and for thousands of years the link between infection and poor nutrition has been well recognized. Today this threat is as widespread as ever, and there are approximately 1 billion undernourished individuals worldwide (3). In the past century, however, the pendulum has also swung in the opposite direction, and now as many if not more people are overweight or obese (4). With the advent of this chronic metabolic overload, a new set of problems and complications at the intersection of metabolism and immunity has emerged, including the obesity-linked inflammatory diseases diabetes, fatty liver disease, airway inflammation, and atherosclerosis (5).

There is now a wealth of evidence indicating close ties between metabolic and immune systems. Among the many reasons to

Nonstandard abbreviations used: AP-1, activator protein-1; DAG, diacylglycerol;

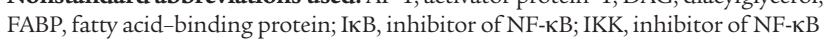
kinase; IRS, insulin receptor substrate; JIP1, JNK-interacting protein-1; LXR, liver X receptor; TLR, Toll-like receptor; TZD, thiazolidinedione.

Conflict of interest: The authors have declared that no conflict of interest exists.

Citation for this article: J. Clin. Invest. 115:1111-1119 (2005).

doi:10.1172/JCI200525102. maintain a healthy weight is the emerging paradigm that metabolic imbalance leads to immune imbalance, with starvation and immunosuppression on one end of the spectrum and obesity and inflammatory diseases on the other end (Figure 1). In this article, we will discuss the molecular and cellular links between metabolism and inflammation, particularly in the context of obesity and diabetes. Common inflammatory mediators, stress responses, and signaling pathways will be highlighted. Finally, we will consider the origin of and the reasons for the inflammatory response in obesity.

\section{Obesity is characterized by inflammation}

Factors at the crossroads of inflammation and metabolic disease. A little more than a decade ago, the first molecular link between inflammation and obesity - TNF- $\alpha$ - was identified when it was discovered that this inflammatory cytokine is overexpressed in the adipose tissues of rodent models of obesity $(6,7)$. As is the case in mice, TNF- $\alpha$ is overproduced in the adipose as well as muscle tissues of obese humans (8-10). Administration of recombinant TNF- $\alpha$ to cultured cells or to whole animals impairs insulin action, and obese mice lacking functional TNF- $\alpha$ or TNF receptors have improved insulin sensitivity compared with wild-type counterparts $(6,11)$. Thus, particularly in experimental models, it is clear that overproduction of TNF- $\alpha$ in adipose tissue is an important feature of obesity and contributes significantly to insulin resistance.

It rapidly became clear, however, that obesity is characterized by a broad inflammatory response and that many inflammatory mediators exhibit patterns of expression and/or impact insulin action in a manner similar to that of TNF- $\alpha$ during obesity, in animals ranging from mice and cats to humans (12-14). Transcriptional profiling studies have revealed that inflammatory and stress-response genes are among the most abundantly regulated gene sets in adipose tissue of obese animals (15-17). A list of many of these genes, which have been identified through a variety of approaches, is provided in Table 1.

In addition to inflammatory cytokines regulating metabolic homeostasis, molecules that are typical of adipocytes, with well-established metabolic functions, can regulate the immune response. Leptin is one such hormone that plays important roles in both adaptive and innate immunity, and both mice and humans lacking leptin function exhibit impaired immunity (18-20). Indeed, reduced leptin levels may be responsible, at least in part, for immunosuppression associated with starvation, as leptin administration has been shown to reverse the immunosuppression of mice starved for 48 hours (21). Adiponectin, resistin, and visfatin are 


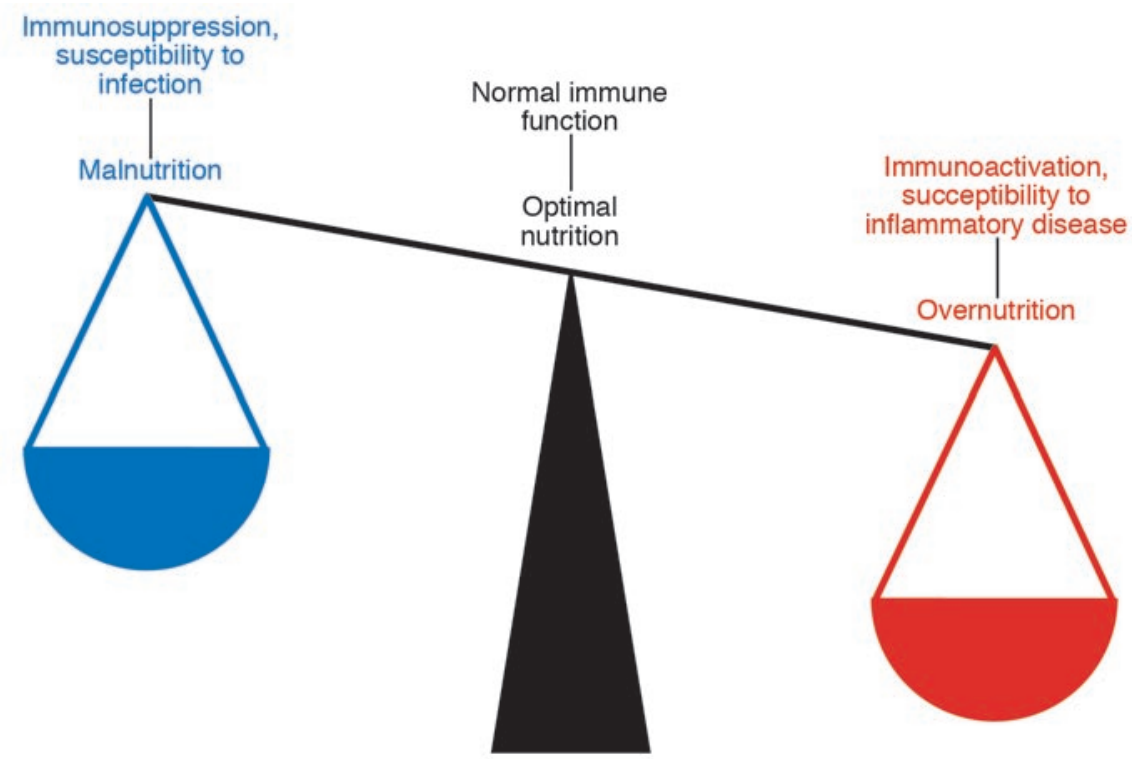

\section{Figure 1}

Metabolism and immunity are closely linked. Both overnutrition and undernutrition have implications for immune function. Starvation and malnutrition can suppress immune function and increase susceptibility to infections. Obesity is associated with a state of aberrant immune activity and increasing risk for associated inflammatory diseases, including atherosclerosis, diabetes, airway inflammation, and fatty liver disease. Thus, optimal nutritional and metabolic homeostasis is an important part of appropriate immune function and good health. also examples of molecules with immunological activity that are produced in adipocytes (22-26).

Finally, lipids themselves also participate in the coordinate regulation of inflammation and metabolism. Elevated plasma lipid levels are characteristic of obesity, infection, and other inflammatory states. Hyperlipidemia in obesity is responsible in part for inducing peripheral tissue insulin resistance and dyslipidemia and contributes to the development of atherosclerosis. It is interesting to note that metabolic changes characteristic of the acute-phase response are also proatherogenic; thus, altered lipid metabolism that is beneficial in the short term in fighting against infection is harmful if maintained chronically (1). The critical importance of bioactive lipids is also evident in their regulation of lipid-targeted signaling pathways through fatty acid-binding proteins (FABPs) and nuclear receptors (see "Regulation of inflammatory pathways," below).

Macrophages and the link between inflammation and metabolism. The high level of coordination of inflammatory and metabolic pathways is highlighted by the overlapping biology and function of macrophages and adipocytes in obesity (Figure 2). Gene expression is highly similar; macrophages express many, if not the majority of "adipocyte" gene products such as the adipocyte/macrophage FABP aP2 (also known as FABP4) and PPAR $\gamma$, while adipocytes can express many "macrophage" proteins such as TNF- $\alpha$, IL-6, and MMPs $(6,27-29)$. Functional capability of these 2 cell types also overlaps. Macrophages can take up and store lipid to become atherosclerotic foam cells. Preadipocytes under some conditions can exhibit phagocytic and antimicrobial properties and appear to even be able to differentiate into macrophages in the right environment, which suggests a potential immune role for preadipocytes $(30,31)$. Furthermore, macrophages and adipocytes colocalize in adipose tissue in obesity. The recent finding that obesity is characterized by macrophage accumulation in white adipose tissue has added another dimension to our understanding of the development of adipose tissue inflammation in obesity $(16,17)$. Macrophages in adipose tissue are likely to contribute to the production of inflammatory mediators either alone or in concert with adipocytes, which suggests a potentially important influence of macrophages in promoting insulin resistance. However, no direct evidence has been offered to establish this connection thus far.

In terms of the immune response, integration between macrophages and adipocytes makes sense, given that both cell types participate in the innate immune response: macrophages in their role as immune cells by killing pathogens and secreting inflammatory cytokines and chemokines; and adipocytes by releasing lipids that may modulate the inflammatory state or participate in neutralization of pathogens. While it is not yet known whether macrophages are drawn to adipose tissue in other inflammatory conditions, it is conceivable that macrophage accumulation in adipose tissue is a feature not only of obesity, but of other inflammatory states as well.

\section{Inflammatory pathways to insulin resistance}

As discussed above, it is now apparent that obesity is associated with a state of chronic, low-grade inflammation, particularly in white adipose tissue. How do inflammatory cytokines and/or fatty acids mediate insulin resistance? How do the stresses of obesity manifest inside of cells? In recent years, much has been learned about the intracellular signaling pathways activated by inflammatory and stress responses and how these pathways intersect with and inhibit insulin signaling.

Insulin affects cells through binding to its receptor on the surface of insulin-responsive cells. The stimulated insulin receptor phosphorylates itself and several substrates, including members of the insulin receptor substrate (IRS) family, thus initiating downstream signaling events $(32,33)$. The inhibition of signaling downstream of the insulin receptor is a primary mechanism through which inflammatory signaling leads to insulin resistance. Exposure of cells to TNF- $\alpha$ or elevated levels of free fatty acids stimulates inhibitory phosphorylation of serine residues of IRS-1 (34-36). This phosphorylation reduces both tyrosine phosphorylation of IRS- 1 in response to insulin and the ability of IRS- 1 to associate with the insulin receptor and thereby inhibits downstream signaling and insulin action $(35,37,38)$.

Recently it has become clear that inflammatory signaling pathways can also become activated by metabolic stresses originating 
Table 1

Factors that mediate the intersection of metabolism and immunity

\begin{tabular}{|c|c|c|c|}
\hline Factors & Metabolic regulation & Effects & Mouse model \\
\hline TNF- $\alpha$ & $\uparrow$ in obesity $(\mathrm{S} 1, \mathrm{~S} 2)^{\mathrm{A}}$ & Promotes insulin resistance (S1, S3) & LOF (S4), GOF (S5) \\
\hline $\mathrm{IL}-6$ & $\uparrow$ in obesity $(\mathrm{S} 6, \mathrm{~S} 7)$ & $\begin{array}{l}\text { Promotes insulin resistance; } \\
\text { central anti-obesity action (S8-S11) }\end{array}$ & LOF (S10), GOF (S11) \\
\hline Leptin & $\uparrow$ in obesity (S12) & $\begin{array}{l}\text { Multiple effects on immune function; } \\
\text { suppresses appetite; } \\
\text { promotes FA oxidation (S12-S14) }\end{array}$ & LOF (S12) \\
\hline Adiponectin & $\downarrow$ in obesity (S15) & $\begin{array}{c}\text { Antiinflammatory; } \\
\text { promotes insulin sensitivity; } \\
\text { stimulates FA oxidation (S15, S16) }\end{array}$ & LOF (S17), GOF (S18, S19) \\
\hline Visfatin & $\uparrow$ in obesity (S20) & Early B cell growth factor; insulin mimetic (S20, S21) & LOF (S20) \\
\hline Resistin & Variable in obesity (S22, S23) & $\begin{array}{l}\text { Induced in endotoxemia/inflammation; } \\
\text { promotes insulin resistance; } \\
\text { regulates fasting blood glucose level (S23-S26) }\end{array}$ & LOF (S24), GOF (S25, S27) \\
\hline IL-1 & $\uparrow$ by hyperglycemia (S28) & $\begin{array}{l}\text { Proinflammatory; } \\
\text { regulates insulin secretion; } \\
\text { involved in central leptin action }(\mathrm{S} 29, \mathrm{~S} 30)\end{array}$ & LOF (S30) \\
\hline IL-1R $\alpha$ & $\uparrow$ in obesity (S31) & Antiinflammatory; opposes leptin action (S29) & \\
\hline $\mathrm{IL}-8$ & $\uparrow$ in obesity (S32) & Proatherogenic (S33, S34) & LOF (S33) \\
\hline IL-10 & $\uparrow$ in obesity; $\downarrow$ in metabolic syndrome (S35) & $\begin{array}{c}\text { Antiinflammatory; } \\
\text { promotes insulin sensitivity (S36) }\end{array}$ & \\
\hline IL-18 & $\uparrow$ in obesity $(\mathrm{S} 37, \mathrm{~S} 38)$ & Proatherogenic (S39, S40) & LOF (S40) GOF (S39) \\
\hline MCP-1 & $\uparrow$ in obesity (S41) & $\begin{array}{l}\text { Proatherogenic; } \\
\text { promotes insulin resistance (S34, S41, S42) }\end{array}$ & LOF (S42) \\
\hline MIF & $\uparrow$ in obesity (S43) & Inhibits macrophage migration & \\
\hline M-CSF & & $\begin{array}{l}\text { Monocyte/macrophage differentiation; } \\
\text { stimulates adipose growth (S44) }\end{array}$ & GOF (S44) \\
\hline TGF- $\beta$ & $\uparrow$ in obesity $(\mathrm{S} 45, \mathrm{~S} 46)$ & $\begin{array}{l}\text { Inhibits adipocyte differentiation and } \\
\text { adipose tissue development; } \\
\text { regulates atherosclerosis (S47-S49) }\end{array}$ & LOF (S50, S51), GOF (S49) \\
\hline Soluble TNFR & $\uparrow$ in obesity (S52-S54) & Proinflammatory & \\
\hline C-reactive protein & $\uparrow$ in obesity (S55, S56) & $\begin{array}{l}\text { Proinflammatory; atherogenic; } \\
\text { risk factor for diabetes (S55, S57-S59) }\end{array}$ & GOF (S57) \\
\hline Haptoglobin & $\uparrow$ in obesity (S60) & Proinflammatory & \\
\hline
\end{tabular}

$\uparrow$, increase; $\downarrow$, decrease; FA, fatty acid; GOF, gain-of-function; IL-1R $\alpha$, IL-1 receptor $\alpha$; LOF, loss-of-function; MCP-1, monocyte chemotactic protein-1; MIF, macrophage migration inhibitory factor; TNFR, TNF receptor. ASee Supplemental References; supplemental material available online with this article; doi:10.1172/JCI200525102DS1.

from inside the cell as well as by extracellular signaling molecules. It has been demonstrated that obesity overloads the functional capacity of the ER and that this ER stress leads to the activation of inflammatory signaling pathways and thus contributes to insulin resistance (39-41). Additionally, increased glucose metabolism can lead to a rise in mitochondrial production of ROS. ROS production is elevated in obesity, which causes enhanced activation of inflammatory pathways $(42,43)$.

Several serine/threonine kinases are activated by inflammatory or stressful stimuli and contribute to inhibition of insulin signaling, including JNK, inhibitor of NF-KB kinase (IKK), and PKC- $\theta$ (44). Again, the activation of these kinases in obesity highlights the overlap of metabolic and immune pathways; these are the same kinases, particularly IKK and JNK, that are activated in the innate immune response by Toll-like receptor (TLR) signaling in response to LPS, peptidoglycan, double-stranded RNA, and other microbial products (45). Hence it is likely that components of TLR signaling pathways will also exhibit strong metabolic activities.

$J N K$. The 3 members of the JNK group of serine/threonine kinases, JNK-1, -2 , and -3 , belong to the MAPK family and regulate multiple activities in development and cell function, in large part through their ability to control transcription by phosphorylating activator protein-1 (AP-1) proteins, including c-Jun and JunB (46). JNK has recently emerged as a central metabolic regulator, playing an important role in the development of insulin resistance in obesity (47). In response to stimuli such as ER stress, cytokines, and fatty acids, JNK is activated, whereupon it associates with and phosphorylates IRS-1 on Ser307, impairing insulin action $(36,39,48)$. In obesity, JNK activity is elevated in liver, muscle, and fat tissues, and loss of JNK1 prevents the development of insulin resistance and diabetes in both genetic and dietary mouse models of obesity (47). Modulation of hepatic JNK1 in adult animals also produces systemic effects on glucose metabolism, which underscores the importance of this pathway in the liver (49). The contribution of the JNK pathway in adipose, muscle, or other tissues to systemic insulin resistance is currently unclear. In addition, a mutation in JNK-interacting protein-1 (JIP1), a protein that binds JNK and regulates its activity, has been identified in diabetic humans (50). The phenotype of the JIP1 loss-of-function model is very 


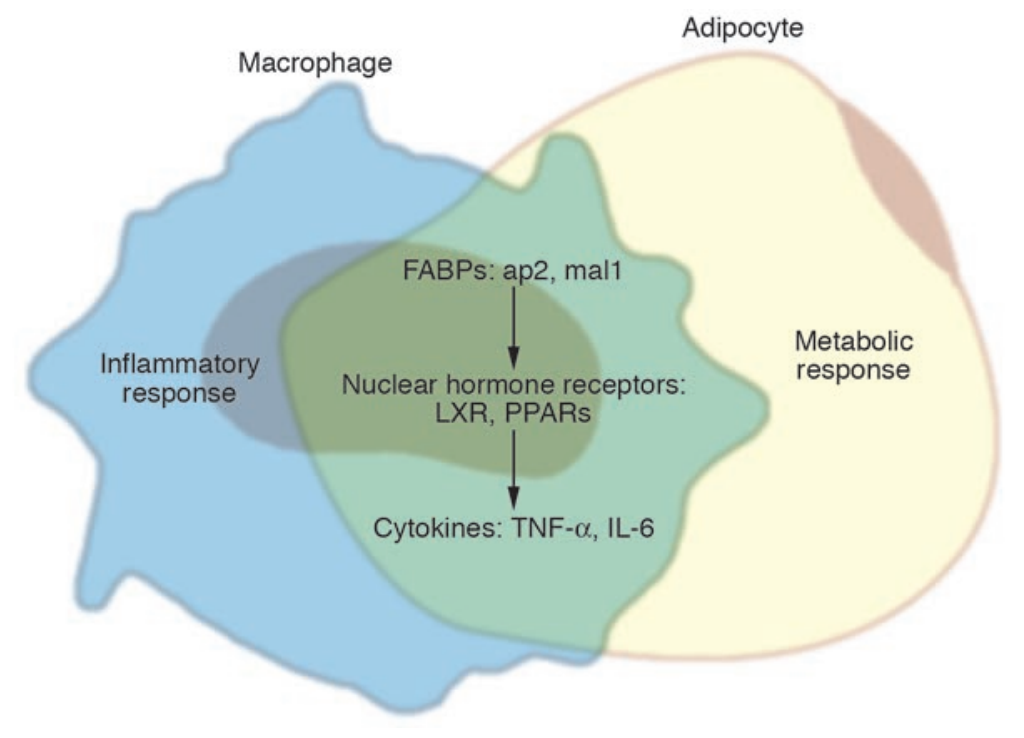

Figure 2

Lipids and inflammatory mediators: integration of metabolic and immune responses in adipocytes and macrophages through shared mechanisms. Under normal conditions, adipocytes store lipids and regulate metabolic homeostasis, and macrophages function in the inflammatory response, although each cell type has the capacity to perform both functions. In obesity, adipose tissue becomes inflamed, both via infiltration of adipose tissue by macrophages and as a result of adipocytes themselves becoming producers of inflammatory cytokines. Inflammation of adipose tissue is a crucial step in the development of peripheral insulin resistance. In addition, in proatherosclerotic conditions such as obesity and dyslipidemia, macrophages accumulate lipid to become foam cells. Adipocytes and macrophages share common features such as expression of cytokines, FABPs, nuclear hormone receptors, and many other factors. As evidenced by genetic loss-of-function models, adipocyte/macrophage FABPs modulate both lipid accumulation in adipocytes and cholesterol accumulation in macrophages, as well as the development of insulin resistance and atherosclerosis. PPAR $\gamma$ and LXR pathways oppose inflammation and promote cholesterol efflux from macrophages and lipid storage in adipocytes. to lipid infusion, high-fat diet, or genetic obesity $(59,60)$. Moreover, inhibition of IKK $\beta$ in human diabetics by high-dose aspirin treatment also improves insulin signaling, although at this dose, it is not clear whether other kinases are also affected (61). Recent studies have also begun to tease out the importance of IKK in individual tissues or cell types to the development of insulin resistance. Activation of IKK in liver and myeloid cells appears to contribute to obesity-induced insulin resistance, though this pathway may not be as important in muscle (62-64).

Other pathways. In addition to serine/ threonine kinase cascades, other pathways contribute to inflammation-induced insulin resistance. For example, at least 3 members of the SOCS family, SOCS1, -3 , and -6 , have been implicated in cytokinemediated inhibition of insulin signaling (65-67). These molecules appear to inhibit insulin signaling either by interfering with IRS- 1 and IRS- 2 tyrosine phosphorylation or by targeting IRS-1 and IRS-2 for proteosomal degradation $(65,68)$. SOCS3 has also been demonstrated to regulate central leptin action, and both whole body reduction in SOCS3 expression $\left(\mathrm{SOCS3}^{+--}\right)$and neural SOCS3 disruption result in resistance to high-fat diet-induced obesity and insulin resistance $(69,70)$.

Inflammatory cytokine stimulation can also lead to induction of iNOS. Overproduction of nitric oxide also appears to similar to that of JNK1 deficiency in mice, with reduced JNK activity and increased insulin sensitivity (51). Interestingly, the JNK2 isoform plays a significant nonredundant role in atherosclerosis (52), though apparently not in type 2 diabetes. Recent studies in mice demonstrate that JNK inhibition in established diabetes or atherosclerosis might be a viable therapeutic avenue for these diseases in humans $(52,53)$.

$P K C$ and IKK. Two other inflammatory kinases that play a large role in counteracting insulin action, particularly in response to lipid metabolites, are IKK and PKC- $\theta$. Lipid infusion has been demonstrated to lead to a rise in levels of intracellular fatty acid metabolites, such as diacylglycerol (DAG) and fatty acyl CoAs. This rise is correlated with activation of PKC- $\theta$ and increased Ser307 phosphorylation of IRS-1 (54). PKC- $\theta$ may impair insulin action by activation of another serine/threonine kinase, IKK $\beta$, or JNK (55). Other PKC isoforms have also been reported to be activated by lipids and may also participate in inhibition of insulin signaling (56).

IKK $\beta$ can impact on insulin signaling through at least 2 pathways. First, it can directly phosphorylate IRS-1 on serine residues $(34,57)$. Second, it can phosphorylate inhibitor of NF-кB (I $\mathrm{B})$, thus activating NF- $\kappa \mathrm{B}$, a transcription factor that, among other targets, stimulates production of multiple inflammatory mediators, including TNF- $\alpha$ and IL-6 (58). Mice heterozygous for IKK $\beta$ are partially protected against insulin resistance due contribute to impairment of both muscle cell insulin action and $\beta$ cell function in obesity $(71,72)$. Deletion of iNOS prevents impairment of insulin signaling in muscle caused by a high-fat diet (72). Thus, induction of SOCS proteins and iNOS represent 2 additional and potentially important mechanisms that contribute to cytokine-mediated insulin resistance. It is likely that additional mechanisms linking inflammation with insulin resistance remain to be uncovered.

\section{Regulation of inflammatory pathways}

Lipids and lipid targets. The role of lipids in metabolic disease is complex. As discussed above, hyperlipidemia leads to increased uptake of fatty acids by muscle cells and production of fatty acid metabolites that stimulate inflammatory cascades and inhibit insulin signaling (54). On the other hand, intracellular lipids can also be antiinflammatory. Ligands of the liver X receptor (LXR) and PPAR families of nuclear hormone receptors are oxysterols and fatty acids, respectively, and activation of these transcription factors inhibits inflammatory gene expression in macrophages and adipocytes, in large part through suppression of NF- $\mathrm{B}$ (73-79).

LXR function is also regulated by innate immune pathways. Signaling from TLRs inhibits LXR activity in macrophages, causing enhanced cholesterol accumulation and accounting, at least in part, for the proatherogenic effects of infection (80). Indeed, lack of MyD88, a critical mediator of TLR signaling, reduces 


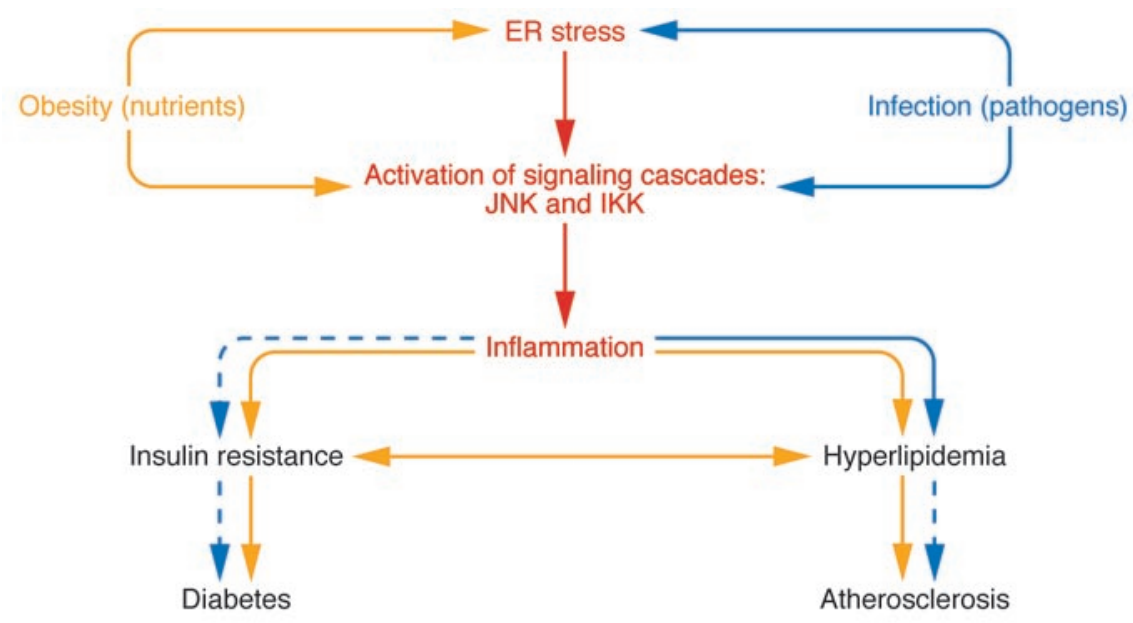

\section{Figure 3}

Nutrient and pathogen sensing or response systems have important overlapping features, and their modulation by obesity or infection can lead to overlapping physiological outcomes. For example, the chronic inflammation of obesity leads to elevated plasma lipid levels and the development of insulin resistance, eventually resulting in fatty liver disease, atherosclerosis, and diabetes. Infection typically leads to a more transient and robust inflammatory response and short-term hyperlipidemia that aids in the resolution of the infection. In some circumstances of chronic infection, however, insulin resistance, diabetes, and atherosclerosis can result. atherosclerosis in apoE $E^{-/-}$mice (81). Interestingly, despite the inhibitory effects of TLR signaling on LXR cholesterol metabolism, LXR appears to be necessary for the complete response of macrophages to infection. In the absence of LXR, macrophages undergo accelerated apoptosis and are thus unable to appropriately respond to infection (82). Unliganded PPAR $\delta$ also seems to have proinflammatory functions, mediated at least in part through its association with the transcriptional repressor B cell lymphoma 6 (BCL-6) (83).

The activity of these lipid ligands is influenced by cytosolic FABPs. Animals lacking the adipocyte/macrophage FABPs ap2 and mal1 are strongly protected against type 2 diabetes and atherosclerosis, a phenotype reminiscent of that of thiazolidinedione-treated (TZD-treated) mice and humans $(27,84,85)$. One mechanism for this phenotype is potentially related to the availability of endogenous ligands for these receptors that stimulate storage of lipids in adipocytes and suppress inflammatory pathways in macrophages (86). In general, it appears that location in the body, the composition of the surrounding cellular environment, and coupling to target signaling pathways are critical for determining whether lipids promote or suppress inflammation and insulin resistance. Accumulation of cholesterol in macrophages promotes atherosclerosis and of lipid in muscle and liver promotes insulin resistance, while, as seen in TZDtreated and FABP-deficient mice, if lipids are forced to remain in adipose tissue, insulin resistance in the context of obesity can be reduced (85). Thus, lipids and their targets clearly play both metabolic and inflammatory roles; however, the functions that they assume are dependent on multiple factors.

Pharmacological manipulation of inflammation. In corroboration of genetic evidence in mice that loss of inflammatory mediators or signaling molecules prevents insulin resistance $(11,47,59)$, pharmacological targeting of inflammatory pathways also improves insulin action. Effective treatment has been demonstrated both with inhibitors of inflammatory kinases and with agonists of relevant transcription factors. As discussed above, salicylates promote insulin signaling by inhibiting inflammatory kinase cascades within the cell. Through inhibition of IKK and possibly other kinases, salicylates are able to improve glucose metabolism in both obese mice and diabetic humans $(53,55,74)$. Targeting of JNK using a synthetic inhibitor and/or an inhibitory peptide has been demonstrated to improve insulin action in obese mice and reduce atherosclerosis in the apoE-deficient rodent model $(52,53)$. These results directly demonstrate the therapeutic potential of JNK inhibitors in diabetes.

Synthetic ligands have been produced to all 3 PPAR isoforms as well as LXR- $\alpha$, though only PPAR $\gamma$ and PPAR $\alpha$ ligands have been approved for clinical treatment $(87,88)$. TZDs, high-affinity ligands of PPAR $\gamma$, which are given clinically as insulin-sensitizing agents, likely improve insulin action through multiple mechanisms, including both activating lipid metabolism and reducing production of inflammatory mediators such as TNF- $\alpha(85,89-93)$. Synthetic PPAR $\alpha$ ligands, fibrates, are used to treat hyperlipidemia. These drugs appear to work predominantly through stimulation of fatty acid oxidation, though they also have antiinflammatory actions that contribute to their effects $(87,94)$. LXR ligands have been demonstrated to improve glucose metabolism in experimental animals (88), and it remains to be seen whether suppression of inflammation contributes to this action.

In targeting inflammation to treat insulin resistance and diabetes, it is possible that seeking inhibitors for individual inflammatory mediators may not be a maximally effective strategy, as other redundant components may be sufficient to continue to propagate inflammatory pathways. For example, targeting individual inflammatory cytokines may not be highly effective, whereas targeting the inflammatory kinases JNK and IKK generates a robust antidiabetic action because these factors integrate signals from multiple inflammatory mediators. On the other hand, if a more central process or mediator can be identified, this may provide an even more attractive target. The ER stress pathway could potentially be one such central process, in that this pathway is able to activate both JNK and IKK; thus, inhibiting the ER stress response through addition of chaperones or other mechanisms could potentially disable both of these arms of the inflammatory response and rescue insulin action (39). It has recently been demonstrated that mice in which the chaperone ORP150 is transgenically or adenovirally overexpressed exhibited reduced ER stress and improved insulin tolerance compared with controls, whereas reduction of the expression of this molecule in liver results in increased ER stress and insulin resistance $(40,41)$.

\section{Origin of inflammation in obesity}

While we are now aware of many of the inflammatory factors that mediate insulin resistance and have some understanding of the intracellular pathways involved, there is still much that remains 


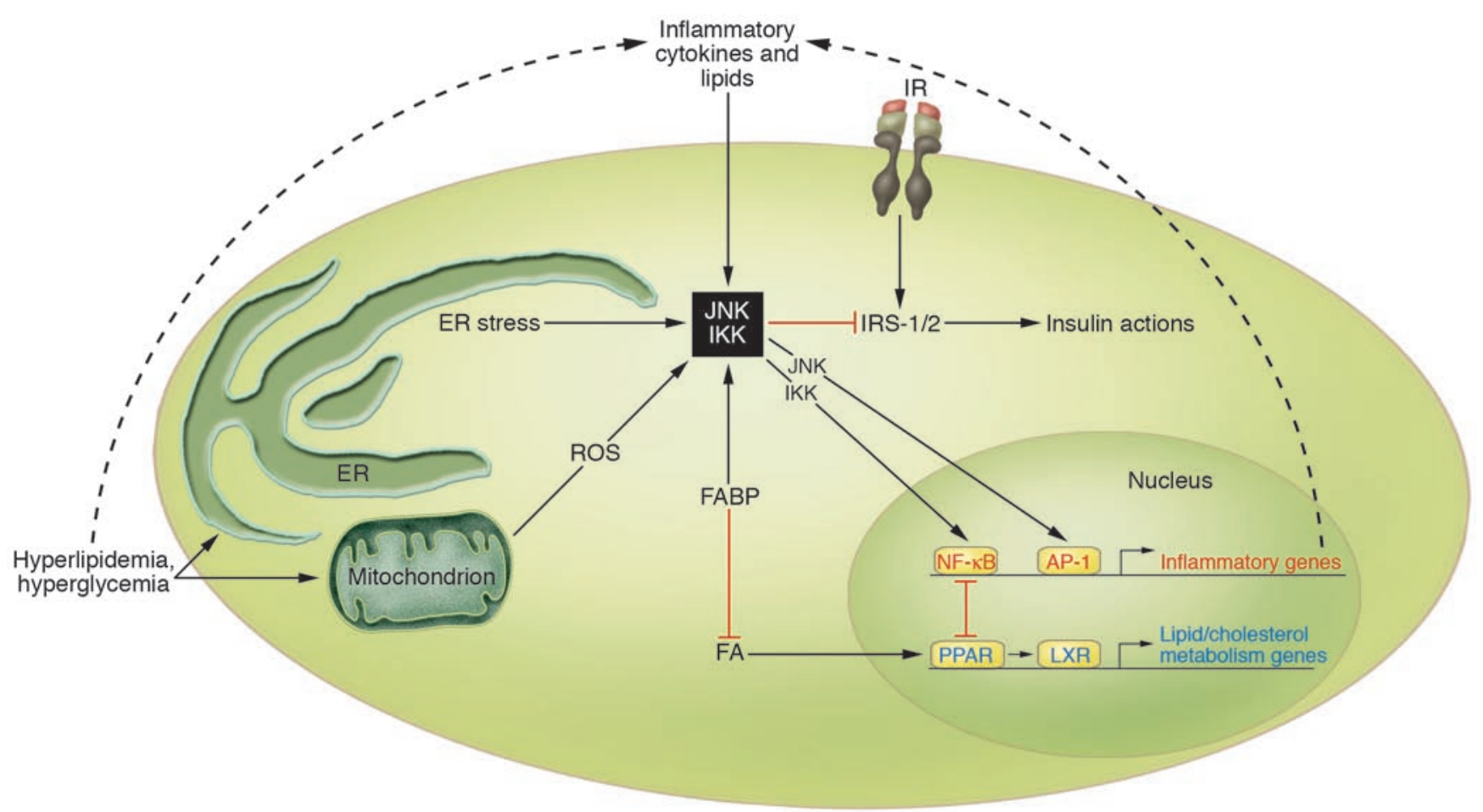

Figure 4

Model of overlapping metabolic and inflammatory signaling and sensing pathways in adipocytes or macrophages. Inflammatory pathways can be initiated by extracellular mediators such as cytokines and lipids or by intracellular stresses such as ER stress or excess ROS production by mitochondria. Signals from all of these mediators converge on inflammatory signaling pathways, including the kinases JNK and IKK. These pathways lead to the production of additional inflammatory mediators through transcriptional regulation as well as to the direct inhibition of insulin signaling. Other pathways such as those mediated through the SOCS proteins and iNOS are also involved in inflammation-mediated inhibition of insulin action. Opposing the inflammatory pathways are transcription factors from the PPAR and LXR families, which promote nutrient transport and metabolism and antagonize inflammatory activity. More proximal regulation is provided by FABPs, which likely sequester ligands of these transcription factors, thus promoting a more inflammatory environment. The absence of FABPs is antiinflammatory. The cell must strike a balance between metabolism and inflammation. In conditions of overnutrition, this becomes a particular challenge, as the very processes required for response to nutrients and nutrient utilization, such as mitochondrial oxidative metabolism and increasing protein synthesis in the ER, can induce the inflammatory response. IR, insulin receptor.

poorly understood. Crucial questions that are currently open regard the initiation of the inflammatory response. Is inflammation the primary event linking obesity with insulin resistance, or does the inflammatory response begin only after the onset of resistance to insulin? How and why does the body initiate an inflammatory response to obesity? Does obesity per se induce an inflammatory response, or is inflammation initiated as a secondary event by hyperlipidemia or hyperglycemia?

In reviewing the facts, it is fairly clear that obesity promotes states of both chronic low-grade inflammation and insulin resistance. However, even in the absence of obesity, infusion of animals with inflammatory cytokines or lipids can cause insulin resistance (54). Additionally, humans with some other chronic inflammatory conditions are at increased risk for diabetes; for example, about one-third of patients with chronic hepatitis $\mathrm{C}$ develop type 2 diabetes, and elevated TNF- $\alpha$ levels are implicated in this link $(95,96)$. Rheumatoid arthritis also predisposes patients to diabetes and particularly cardiovascular disease, and some evidence indicates a link between inflammatory lung diseases and risk of cardiovascular disease and diabetes (97-99). Finally, removal of inflammatory mediators or pathway components, such as TNF- $\alpha$, JNK, and IKK, protects against insulin resistance in obese mouse models, and treatment of humans with drugs that target these pathways, such as salicylates, improves insulin sensitivity $(6,11,47,59,61)$. Thus, the available evidence strongly suggests that type 2 diabetes is an inflammatory disease and that inflammation is a primary cause of obesity-linked insulin resistance, hyperglycemia, and hyperlipidemia rather than merely a consequence (Figure 3 ).

But how does the inflammatory response begin? Though this question cannot currently be answered, we can suggest some reasonable speculations based on the available data. It seems likely that the inflammatory response is initiated in the adipocytes themselves, as they are the first cells affected by the development of obesity, or potentially in neighboring cells that may be affected by adipose growth. How might expanding adipocytes trigger an inflammatory response?

One mechanism that, based on newly emerging data, appears to be of central importance is the activation of inflammatory pathways by ER stress. Obesity generates conditions that increase the demand on the ER (39-41). This is particularly the case for adipose tissue, which undergoes severe changes in tissue architecture, increases in protein and lipid synthesis, and perturbations in intracellular nutrient and energy fluxes. In both cultured cells and whole animals, ER stress leads to activation 
of JNK and thus contributes to insulin resistance (39). Interestingly, ER stress also activates IKK and thus may represent a common mechanism for the activation of these 2 important signaling pathways (100).

A second mechanism that may be relevant in the initiation of inflammation in obesity is oxidative stress. Due to increased delivery of glucose to adipose tissue, endothelial cells in the fat pad may take up increasing amounts of glucose through their constitutive glucose transporters. Increased glucose uptake by endothelial cells in hyperglycemic conditions causes excess production of ROS in mitochondria, which inflicts oxidative damage and activates inflammatory signaling cascades inside endothelial cells (101). Endothelial injury in the adipose tissue might attract inflammatory cells such as macrophages to this site and further exacerbate the local inflammation. Hyperglycemia also stimulates ROS production in adipocytes, which leads to increased production of proinflammatory cytokines (42).

\section{Why inflammation?}

Perhaps one of the most difficult questions to answer is why obesity elicits an inflammatory response. Why, if the ability to store excess energy has been preserved through the course of evolution, does the body react in a manner that is harmful to itself? We hypothesize that this reaction is tied to the interdependency of metabolic and immune pathways.

Could obesity-induced inflammation simply be a side effect of this interaction that was never selected against since chronic obesity and its associated disorders have been so rare over time for people in their reproductive years? Perhaps the stresses of obesity are similar enough to the stresses of an infection that the body reacts to obesity as it would to an infection. For example, in both infection and obesity, intracellular stress pathways such as the JNK and IKK-NF- $\mathrm{KB}$ pathways are activated. Could these pathways be activated by similar mechanisms in both conditions? One mechanism that appears to be critical for initiation of this response in both situations is ER stress. During viral infection, stress pathways are activated by an excess of viral proteins in the ER (102). Similarly, the demands of obesity also result in an overloaded ER and activation of these pathways (39). Another scenario might be related to the capturing of components of the insulin-signaling pathway by microorganisms. Some pathogens activate host intracellular signaling cascades, including the PI3KAkt pathway, which is also critical for insulin signaling (102). Perhaps in a situation in which this pathway becomes overstimulated by an increased need to take up glucose, the cell begins to interpret the signal as an indication of infection and responds by resisting the anabolic insulin signal and instead activating catabolic and inflammatory pathways.

On the other hand, perhaps the inflammatory response to obesity is not simply an undesirable byproduct, but rather a homeostatic mechanism to prevent the organism from reaching a point at which excess fat accumulation impairs mobility or otherwise diminishes fitness. Lipid storage and accumulation of fat weight require anabolic processes, exemplified by insulin action, whereas inflammation stimulates catabolism, including lipolysis from adipocytes. It is conceivable that mechanisms such as the activation of catabolism via inflammation (and hence resistance to anabolic signals) may be an attempt to keep body weight within acceptable bounds. While there is no available experimental evidence that addresses the role of low-grade inflammation in such homeostasis, some support for this idea can be seen in findings that experimentally induced local inflammation or insulin resistance in adipose tissue, such as that in adipose-specific insulin receptor knockout mice and adipose-specific TNF transgenic mice, is metabolically favorable, resulting in a lean phenotype and systemic insulin sensitivity $(103,104)$.

\section{Conclusions}

Our understanding of the characteristics of inflammation in obesity and the mechanisms by which this inflammation contributes to insulin resistance has been increasing rapidly over the last decade, such that we can now suggest a synthesized model (Figure 4). While it is clear that inhibition of insulin receptor signaling pathways is a central mechanism through which inflammatory and stress responses mediate insulin resistance, it is likely that other relevant pathways, molecules, and alternative mechanisms involved in this interaction have yet to be uncovered. Of particular interest is the role of alterations in mitochondrial function in diabetes. While we did not cover this topic in this article, the reader is referred to an excellent recent review for more information (105).

Another important question is whether genetic differences can predispose some individuals to inflammation-mediated insulin resistance. Several studies have reported associations between diabetes and polymorphisms in the promoters of TNF- $\alpha$ and IL- 6 (106-109). The most well-accepted polymorphism associated with type 2 diabetes is found in the gene encoding PPAR $\gamma(110)$. As it is a transcription factor with some antiinflammatory activities, such as suppressing the production of TNF- $\alpha$, one could imagine how altered activity of PPAR $\gamma$ could affect susceptibility to inflammation in obesity. Similarly, genetic variations in the FABP, JNK, IKK, or ER stress pathways or any other loci that modulate the extent of inflammation and consequently insulin resistance could define the risk of individuals for developing metabolic complication of obesity.

Finally, in addition to diabetes and cardiovascular disease, inflammation is also known to be important for linking obesity to airway inflammation and asthma, fatty liver disease, and possibly cancer and other pathologies. Understanding the mechanisms leading from obesity to inflammation will have important implications for the design of novel therapies to reduce the morbidity and mortality of obesity through the prevention of its associated chronic inflammatory disorders.

\section{Acknowledgments}

We are grateful to the members of the Hotamisligil laboratory for their contributions. Research in the Hotamisligil laboratory has been supported by the NIH, the American Diabetes Association, and the Pew and Sandler Foundations. We regret the omission of many important references by our colleagues in the field due to space limitations.

Note: References S1-S60 are available online with this article; doi:10.1172/JCI200525102DS1.

Address correspondence to: Gökhan S. Hotamisligil, Department of Genetics and Complex Diseases, Harvard School of Public Health, 665 Huntington Avenue, Boston, Massachusetts 02115, USA. Phone: (617) 432-1950; Fax: (617) 432-1941; E-mail: ghotamis@hsph.harvard.edu. 
1. Khovidhunkit, W., et al. 2004. Effects of infection and inflammation on lipid and lipoprotein metabolism: mechanisms and consequences to the host [review]. J. Lipid Res. 45:1169-1196.

2. Chandra, R.K. 1996. Nutrition, immunity and infection: from basic knowledge of dietary manipulation of immune responses to practical application of ameliorating suffering and improving survival. Proc. Natl. Acad. Sci. U. S. A. 93:14304-14307.

3. Blackburn, G.L. 2001. Pasteur's Quadrant and malnutrition. Nature. 409:397-401.

4. Cummings, D.E., and Schwartz, M.W. 2003. Genetics and pathophysiology of human obesity. Annu. Rev. Med. 54:453-471.

5. Hotamisligil, G.S. 2004. Inflammation, TNFalpha, and insulin resistance. In Diabetes mellitus: a fundamental and clinical text. D.T.S. LeRoith and J.M. Olefsky, editors. 3rd edition. Lippincott, Williams and Wilkins. New York, New York, USA. 953-962.

6. Hotamisligil, G.S., Shargill, N.S., and Spiegelman, B.M. 1993. Adipose expression of tumor necrosis factor-alpha: direct role in obesity-linked insulin resistance. Science. 259:87-91.

7. Sethi, J.K., and Hotamisligil, G.S. 1999. The role of TNF alpha in adipocyte metabolism. Semin. Cell Dev. Biol. 10:19-29.

8. Hotamisligil, G.S., et al. 1995. Increased adipose tissue expression of tumor necrosis factor-alpha in human obesity and insulin resistance. J. Clin. Invest. 95:2409-2415.

9. Kern, P.A., et al. 1995. The expression of tumor necrosis factor in human adipose tissue. Regulation by obesity, weight loss, and relationship to lipoprotein lipase. J. Clin. Invest. 95:2111-2119.

10. Saghizadeh, M., et al. 1996. The expression of TNF alpha by human muscle. Relationship to insulin resistance. J. Clin. Invest. 97:1111-1116.

11. Uysal, K.T., et al. 1997. Protection from obesityinduced insulin resistance in mice lacking TNFalpha function. Nature. 389:610-614.

12. Pickup, J.C. 2004. Inflammation and activated innate immunity in the pathogenesis of type 2 diabetes. Diabetes Care. 27:813-823.

13. Dandona, P., Aljada, A., and Bandyopadhyay, A. 2004. Inflammation: the link between insulin resistance, obesity and diabetes. Trends Immunol. 25:4-7.

14. Miller, C., et al. 1998. Tumor necrosis factor-alpha levels in adipose tissue of lean and obese cats. J. Nutr. 128(Suppl. 12):2751S-2752S.

15. Soukas, A., et al. 2000. Leptin-specific patterns of gene expression in white adipose tissue. Genes Dev. 14:963-980.

16. Weisberg, S.P., et al. 2003. Obesity is associated with macrophage accumulation in adipose tissue. J. Clin. Invest. 112:1796-1808. doi:10.1172/ JCI200319246.

17. Xu, H., et al. 2003. Chronic inflammation in fat plays a crucial role in the development of obesity-related insulin resistance. J. Clin. Invest. 112:1821-1830. doi:10.1172/JCI200319451.

18. Fernandes, G., et al. 1978. Immune response in the mutant diabetic C57BL/Ks-dt+ mouse. Discrepancies between in vitro and in vivo immunological assays. J. Clin. Invest. 61:243-250.

19. Farooqi, I.S., et al. 2002. Beneficial effects of leptin on obesity, T cell hyporesponsiveness, and neuroendocrine/metabolic dysfunction of human congenital leptin deficiency. J. Clin. Invest. 110:1093-1103. doi:10.1172/JCI200215693.

20. Chandra, R.K. 1980. Cell-mediated immunity in genetically obese C57BL/6J ob/ob) mice. Am. J. Clin. Nutr. 33:13-16.

21. Lord, G.M., et al. 1998. Leptin modulates the T-cell immune response and reverses starvation-induced immunosuppression. Nature. 394:897-901.

22. Berg, A.H., Combs, T.P., and Scherer, P.E. 2002. ACRP30/adiponectin: an adipokine regulating glucose and lipid metabolism. Trends Endocrinol. Metab. 13:84-89.

23. Ouchi, N., et al. 2003. Obesity, adiponectin and vascular inflammatory disease. Curr. Opin. Lipidol. 14:561-566.

24. Steppan, C.M., and Lazar, M.A. 2004. The current biology of resistin. J. Intern. Med. 255:439-447.

25. Lehrke, M., et al. 2004. An inflammatory cascade leading to hyperresistinemia in humans. PLOS Med. 1:e45. doi:10.1371/journal.pmed.0010045.

26. Fukuhara, A., et al. 2005. Visfatin: a protein secreted by visceral fat that mimics the effects of insulin. Science. 307:426-430.

27. Makowski, L., et al. 2001. Lack of macrophage fatty-acid-binding protein aP2 protects mice deficient in apolipoprotein $\mathrm{E}$ against atherosclerosis. Nat. Med. 7:699-705.

28. Tontonoz, P., et al. 1998. PPARgamma promotes monocyte/macrophage differentiation and uptake of oxidized LDL. Cell. 93:241-252.

29. Bouloumie, A., et al. 2001. Adipocyte produces matrix metalloproteinases 2 and 9: involvement in adipose differentiation. Diabetes. 50:2080-2086.

30. Cousin, B., et al. 1999. A role for preadipocytes as macrophage-like cells. FASEB J. 13:305-312.

31. Charriere, G., et al. 2003. Preadipocyte conversion to macrophage. Evidence of plasticity. J. Biol. Chem. 278:9850-9855.

32. White, M.F. 1997. The insulin signalling system and the IRS proteins. Diabetologia. 40(Suppl. 2):S2-S17.

33. Saltiel, A.R., and Pessin, J.E. 2002. Insulin signaling pathways in time and space. Trends Cell Biol. 12:65-71.

34. Yin, M.J., Yamamoto, Y., and Gaynor, R.B. 1998. The anti-inflammatory agents aspirin and salicylate inhibit the activity of I(kappa)B kinase-beta. Nature. 396:77-80.

35. Hotamisligil, G.S., et al. 1996. IRS-1-mediated inhibition of insulin receptor tyrosine kinase activity in TNF-alpha- and obesity-induced insulin resistance. Science. 271:665-668.

36. Aguirre, V., et al. 2000. The c-Jun NH(2)-terminal kinase promotes insulin resistance during association with insulin receptor substrate-1 and phosphorylation of Ser(307). J. Biol. Chem. 275:9047-9054.

37. Aguirre, V., et al. 2002. Phosphorylation of Ser307 in insulin receptor substrate-1 blocks interactions with the insulin receptor and inhibits insulin action. J. Biol. Chem. 277:1531-1537.

38. Paz, K., et al. 1997. A molecular basis for insulin resistance. Elevated serine/threonine phosphorylation of IRS- 1 and IRS- 2 inhibits their binding to the juxtamembrane region of the insulin receptor and impairs their ability to undergo insulin-induced tyrosine phosphorylation. J. Biol. Chem. 272:29911-29918.

39. Ozcan, U., et al. 2004. Endoplasmic reticulum stress links obesity, insulin action, and type 2 diabetes. Science. 306:457-461.

40. Nakatani, Y., et al. 2005. Involvement of endoplas$\mathrm{mic}$ reticulum stress in insulin resistance and diabetes. J. Biol. Chem. 280:847-851.

41. Ozawa, K., et al. 2005. The endoplasmic reticulum chaperone improves insulin resistance in type 2 diabetes. Diabetes. 54:657-663.

42. Lin, Y., et al. 2005. The hyperglycemia-induced inflammatory response in adipocytes: the role of reactive oxygen species. J. Biol. Chem. 280:4617-4626.

43. Furukawa, S., et al. 2004. Increased oxidative stress in obesity and its impact on metabolic syndrome. J. Clin. Invest. 114:1752-1761. doi:10.1172/ JCI200421625.

44. Zick, Y. 2003. Role of Ser/Thr kinases in the uncoupling of insulin signaling. Int. J. Obes. Relat. Metab. Disord. 27(Suppl. 3):S56-S60.

45. Medzhitov, R. 2001. Toll-like receptors and innate immunity. Nat. Rev. Immunol. 1:135-145.
46. Davis, R.J. 2000. Signal transduction by the JNK group of MAP kinases. Cell. 103:239-252.

47. Hirosumi, J., et al. 2002. A central role for JNK in obesity and insulin resistance. Nature. 420:333-336.

48. Gao, Z., et al. 2004. Inhibition of insulin sensitivity by free fatty acids requires activation of multiple serine kinases in 3T3-L1 adipocytes. Mol. Endocrinol. 18:2024-2034

49. Nakatani, Y., et al. 2004. Modulation of the JNK pathway in liver affects insulin resistance status. J. Biol. Chem. 279:45803-45809.

50. Waeber, G., et al. 2000. The gene MAPK8IP1, encoding islet-brain-1, is a candidate for type 2 diabetes. Nat. Genet. 24:291-295.

51. Jaeschke, A., Czech, M.P., and Davis, R.J. 2004. An essential role of the JIP1 scaffold protein for JNK activation in adipose tissue. Genes Dev. 18:1976-1980.

52. Ricci, R., et al. 2004. Requirement of JNK2 for scavenger receptor A-mediated foam cell formation in atherogenesis. Science. 306:1558-1561.

53. Kaneto, H., et al. 2004. Possible novel therapy for diabetes with cell-permeable JNK-inhibitory peptide. Nat. Med. 10:1128-1132.

54. Yu, C., et al. 2002. Mechanism by which fatty acids inhibit insulin activation of insulin receptor substrate-1 (IRS-1)-associated phosphatidylinositol 3 -kinase activity in muscle. J. Biol. Chem. 277:50230-50236.

55. Perseghin, G., Petersen, K., and Shulman, G.I. 2003. Cellular mechanism of insulin resistance: potential links with inflammation. Int. J. Obes. Relat. Metab. Disord. 27(Suppl. 3):S6-S11.

56. Schmitz-Peiffer, C. 2002. Protein kinase C and lipid-induced insulin resistance in skeletal muscle. Ann. N. Y. Acad. Sci. 967:146-157.

57. Gao, Z., et al. 2002. Serine phosphorylation of insulin receptor substrate 1 by inhibitor kappa B kinase complex. J. Biol. Chem. 277:48115-48121.

58. Shoelson, S.E., Lee, J., and Yuan, M. 2003. Inflammation and the IKK beta/I kappa B/NF-kappa B axis in obesity- and diet-induced insulin resistance. Int. J. Obes. Relat. Metab. Disord. 27(Suppl. 3):S49-S52.

59. Yuan, M., et al. 2001. Reversal of obesity- and dietinduced insulin resistance with salicylates or targeted disruption of Ikkbeta. Science. 293:1673-1677.

60. Kim, J.K., et al. 2001. Prevention of fat-induced insulin resistance by salicylate. J. Clin. Invest. 108:437-446. doi:10.1172/JCI200111559.

61. Hundal, R.S., et al. 2002. Mechanism by which high-dose aspirin improves glucose metabolism in type 2 diabetes. J. Clin. Invest. 109:1321-1326. doi:10.1172/JCI200214955.

62. Cai, D., et al. 2005. Local and systemic insulin resistance resulting from hepatic activation of IKK-beta and NF-kappaB. Nat. Med. 11:183-190.

63. Arkan, M.C., et al. 2005. IKK-beta links inflammation to obesity-induced insulin resistance. Nat. Med. 11:191-198

64. Rohl, M., et al. 2004. Conditional disruption of IkappaB kinase 2 fails to prevent obesity-induced insulin resistance. J. Clin. Invest. 113:474-481. doi:10.1172/JCI200418712.

65. Rui, L., et al. 2002. SOCS-1 and SOCS-3 block insulin signaling by ubiquitin-mediated degradation of IRS1 and IRS2. J. Biol. Chem. 277:42394-42398.

66. Mooney, R.A., et al. 2001. Suppressors of cytokine signaling- 1 and -6 associate with and inhibit the insulin receptor. A potential mechanism for cytokine-mediated insulin resistance. J. Biol. Chem. 276:25889-25893.

67. Emanuelli, B., et al. 2001. SOCS-3 inhibits insulin signaling and is up-regulated in response to tumor necrosis factor-alpha in the adipose tissue of obese mice. J. Biol. Chem. 276:47944-47949.

68. Ueki, K., Kondo, T., and Kahn, C.R. 2004. Suppressor of cytokine signaling 1 (SOCS-1) and SOCS-3 cause insulin resistance through inhibition of tyrosine phosphorylation of insulin receptor substrate 
proteins by discrete mechanisms. Mol. Cell Biol. 24:5434-5446.

69. Mori, H., et al. 2004. Socs3 deficiency in the brain elevates leptin sensitivity and confers resistance to diet-induced obesity. Nat. Med. 10:739-743.

70. Howard, J.K., et al. 2004. Enhanced leptin sensitivity and attenuation of diet-induced obesity in mice with haploinsufficiency of Socs3. Nat. Med. 10:734-738.

71. Shimabukuro, M., et al. 1997. Role of nitric oxide in obesity-induced beta cell disease. J. Clin. Invest. 100:290-295.

72. Perreault, M., and Marette, A. 2001. Targeted disruption of inducible nitric oxide synthase protects against obesity-linked insulin resistance in muscle. Nat. Med. 7:1138-1143.

73. Seo, J.B., et al. 2004. Activated liver X receptors stimulate adipocyte differentiation through induction of peroxisome proliferator-activated receptor gamma expression. Mol. Cell. Biol. 24:3430-3444.

74. Moller, D.E., and Berger, J.P. 2003. Role of PPARs in the regulation of obesity-related insulin sensitivity and inflammation. Int. J. Obes. Relat. Metab. Disord. 27(Suppl. 3):S17-S21.

75. Joseph, S.B., et al. 2003. Reciprocal regulation of inflammation and lipid metabolism by liver $\mathrm{X}$ receptors. Nat. Med. 9:213-219.

76. Jiang, C., Ting, A.T., and Seed, B. 1998. PPARgamma agonists inhibit production of monocyte inflammatory cytokines. Nature. 391:82-86.

77. Chawla, A., et al. 2001. PPAR-gamma dependent and independent effects on macrophage-gene expression in lipid metabolism and inflammation. Nat. Med. 7:48-52.

78. Chawla, A., et al. 2001. A PPAR gamma-LXR-ABCA1 pathway in macrophages is involved in cholesterol efflux and atherogenesis. Mol. Cell. 7:161-171.

79. Daynes, R.A., and Jones, D.C. 2002. Emerging roles of PPARs in inflammation and immunity. Nat. Rev. Immunol. 2:748-759.

80. Castrillo, A., et al. 2003. Crosstalk between LXR and toll-like receptor signaling mediates bacterial and viral antagonism of cholesterol metabolism. Mol. Cell. 12:805-816.

81. Bjorkbacka, H., et al. 2004. Reduced atherosclerosis in MyD88-null mice links elevated serum cholesterol levels to activation of innate immunity signaling pathways. Nat. Med. 10:416-421.

82. Joseph, S.B., et al. 2004. LXR-dependent gene expression is important for macrophage survival and the innate immune response. Cell. 119:299-309.

83. Lee, C.H., et al. 2003. Transcriptional repression of atherogenic inflammation: modulation by
PPARdelta. Science. 302:453-457

84. Maeda, K., et al. 2005. Adipocyte/macrophage fatty acid binding proteins control integrated metabolic responses in obesity and diabetes. Cell Metabolism. 1:107-119.

85. Spiegelman, B.M. 1998. PPAR-gamma: adipogenic regulator and thiazolidinedione receptor. Diabetes. 47:507-514.

86. Makowski, L., and Hotamisligil, G.S. 2004. Fatty acid binding proteins - the evolutionary crossroads of inflammatory and metabolic responses. J. Nutr. 134:2464S-2468S

87. Lee, C.H., Olson, P., and Evans, R.M. 2003. Minireview: lipid metabolism, metabolic diseases, and peroxisome proliferator-activated receptors. Endocrinology. 144:2201-2207.

88. Laffitte, B.A., et al. 2003. Activation of liver X receptor improves glucose tolerance through coordinate regulation of glucose metabolism in liver and adipose tissue. Proc. Natl. Acad. Sci. U. S. A. 100:5419-5424.

89. Wellen, K.E., et al. 2004. Interaction of tumor necrosis factor-alpha- and thiazolidinedione-regulated pathways in obesity. Endocrinology. 145:2214-2220.

90. Peraldi, P., Xu, M., and Spiegelman, B.M. 1997. Thiazolidinediones block tumor necrosis factoralpha-induced inhibition of insulin signaling. J. Clin. Invest. 100:1863-1869.

91. Ruan, H., Pownall, H.J., and Lodish, H.F. 2003. Troglitazone antagonizes tumor necrosis factor-alpha-induced reprogramming of adipocyte gene expression by inhibiting the transcriptional regulatory functions of NF-kappaB. J. Biol. Chem. 278:28181-28192.

92. Miles, P.D., et al. 1997. TNF-alpha-induced insulin resistance in vivo and its prevention by troglitazone. Diabetes. 46:1678-1683.

93. Lehmann, J.M., et al. 1995. An antidiabetic thiazolidinedione is a high affinity ligand for peroxisome proliferator-activated receptor gamma (PPAR gamma). J. Biol. Chem. 270:12953-12956.

94. Staels, B., et al. 1998. Activation of human aortic smooth-muscle cells is inhibited by PPARalpha but not by PPARgamma activators. Nature. 393:790-793.

95. Knobler, H., et al. 2003. Tumor necrosis factor-alphainduced insulin resistance may mediate the hepatitis $\mathrm{C}$ virus-diabetes association. Am. J. Gastroenterol. 98:2751-2756.

96. Bahtiyar, G., et al. 2004. Association of diabetes and hepatitis $\mathrm{C}$ infection: epidemiologic evidence and pathophysiologic insights. Curr. Diab. Rep. 4:194-198.
97. Iribarren, C., Tolstykh, I.V., and Eisner, M.D. 2004. Are patients with asthma at increased risk of coronary heart disease? Int. J. Epidemiol. 33:743-748.

98. Rana, J.S., et al. 2004. Chronic obstructive pulmonary disease, asthma, and risk of type 2 diabetes in women. Diabetes Care. 27:2478-2484.

99. Sattar, N., et al. 2003. Explaining how "highgrade" systemic inflammation accelerates vascular risk in rheumatoid arthritis. Circulation. 108:2957-2963.

100.Hung, J.H., et al. 2004. Endoplasmic reticulum stress stimulates the expression of cyclooxygenase-2 through activation of NF-kappaB and pp38 mitogen-activated protein kinase. J. Biol. Chem. 279:46384-46392.

101. Brownlee, M. 2001. Biochemistry and molecular cell biology of diabetic complications. Nature. 414:813-820.

102.Hatada, E.N., Krappmann, D., and Scheidereit, C. 2000. NF-kappaB and the innate immune response. Curr. Opin. Immunol. 12:52-58.

103. Bluher, M., et al. 2002. Adipose tissue selective insulin receptor knockout protects against obesity and obesity-related glucose intolerance. Dev. Cell. 3:25-38.

104.Xu, H., et al. 2002. Exclusive action of transmembrane TNF alpha in adipose tissue leads to reduced adipose mass and local but not systemic insulin resistance. Endocrinology. 143:1502-1511.

105.Lowell, B.B., and Shulman, G.I. 2005. Mitochondrial dysfunction and type 2 diabetes. Science. 307:384-387.

106.Vozarova, B., et al. 2003. The interleukin-6 (-174) $\mathrm{G} / \mathrm{C}$ promoter polymorphism is associated with type- 2 diabetes mellitus in Native Americans and Caucasians. Hum. Genet. 112:409-413.

107. Dalziel, B., et al. 2002. Association of the TNFalpha $-308 \mathrm{G} / \mathrm{A}$ promoter polymorphism with insulin resistance in obesity. Obes. Res. 10:401-407.

108. Costa, A., et al. 2003. Lower rate of tumor necrosis factor-alpha -863A allele and higher concentration of tumor necrosis factor-alpha receptor 2 in firstdegree relatives of subjects with type 2 diabetes. Metabolism. 52:1068-1071.

109. Furuta, M., et al. 2002. Relationship of the tumor necrosis factor-alpha $-308 \mathrm{~A} / \mathrm{G}$ promoter polymorphism with insulin sensitivity and abdominal fat distribution in Japanese patients with type 2 diabetes mellitus. Diabetes Res. Clin. Pract. 56:141-145.

110.Florez, J.C., Hirschhorn, J., and Altshuler, D. 2003. The inherited basis of diabetes mellitus: implications for the genetic analysis of complex traits. Annu. Rev. Genomics Hum. Genet. 4:257-291. 